\title{
Contribution of aroma compounds to the antioxidant properties of roasted white yam (Dioscorea rotundata)
}

\author{
Ola Lasekan* ${ }^{*}$ and Li Shing Teoh
}

\begin{abstract}
Background: The aroma chemistry and the contribution of the aroma compounds to the anti-oxidative properties of roasted yam have yet to be characterized. The growing popularity of roasted yam in regions where they are being consumed calls for a concerted effort to elucidate their aroma chemistry as well as their anti-oxidative properties.

Results: The aroma compounds in roasted white yam (Dioscorea rotundata) were isolated and identified using static headspace-gas chromatography-mass spectrometry (SH-GC-MS) and gas chromatography-olfactometry (GC-O). In addition, the anti-oxidative activities of the most abundant volatile heterocyclic compounds (2 pyrroles, 4 furans and 3 pyrazines) were evaluated on their inhibitory effect towards the oxidation of hexanal for a period of 30 days. Twenty-nine aroma-active compounds with a flavour dilution (FD) factor range of 2-256 and an array of odour notes were obtained. Among them, the highest odour activities (FD $\geq 128$ ) factors were determined for 2-acetyl furan and 2-acetylpyrrole. Other compounds with significant FD factors $\geq 32$ were; 2-methylpyrazine, ethyl furfural, and 5-hydroxy methyl furfural.

Conclusion: Results of the anti-oxidative activity showed that the pyrroles exhibited the greatest antioxidant activity among all the tested heterocyclic compounds. This was followed by the furans and the pyrazines which had the least antioxidant activity.
\end{abstract}

Keywords: Roasted white yam, Aroma-active compounds, Antioxidant activity

\section{Background}

Dioscorea (yam) species are made up of 613 genera of tuberous climbing plants [1]. However, only two of these (D. alata and D. cayenennsis Lam. Subspecies, cayenennsis and $D$. cayenennsis Lam subspecies rotundata (Poir) are of economic importance and nourishment to the inhabitants of tropical regions of the world. Yam tuber belongs to the family Dioscoreacea and it's made up of about 600 species [1]. While most of these species are toxic, about ten species are regarded as edible. Of these, the most widely cultivated are; $D$. rotundata (white yam), $D$. cayenennsis Lam (yellow yam) and $D$. alata (water yam). These yam tubers are of primary importance as

\footnotetext{
*Correspondence: olaniny56@gmail.com
}

Department of Food Technology, University Putra Malaysia, 43400

UPM Serdang, Malaysia stable food in West Africa, the Caribbean Islands, South East Asia and tropical America [2]. Apart from providing food security to the growing population in the subSahara African countries, yam serves as a rich source of carbohydrate and contributes to the vitamins and minerals of the people.

Yam is usually consumed in different forms such as; boiled, fried and roasted. Roasted yams hold cultural and social importance in some societies in Nigeria. Roasted yam is produced by roasting yam tubers on a grill above heated charcoal with constant turning until a uniform dark brown colour is achieved [3]. The roasted yam with distinct characteristic aroma is eaten with hot palm oil sauce. While raw yam has only a faint odour, the roasted yam exhibits a characteristic toasty cum cracker-like aroma. Despite the growing popularity of roasted yam, nothing is known of its aroma components. Knowledge 
of food volatile constituents and concentrations has been used in breeding programs [4]. Aroma is cultivar-specific and a study of volatile profile is very important.

A great variety of aroma compounds, most of which are Maillard reaction products (MRPs) are produced during roasting at high temperatures [5]. The MRPs are complex mixtures of different compounds such as; aldehydes, ketones, dicarbonyls, heterocyclic amines, acryl amines, melanoidins and advanced glycation end products (AGEs) [6] formed at the advanced stage of Maillard reaction. Some of the MRPs are known to contribute toasted or roasted flavour to heated foods [7]. Prior to this time, these MRPs were studied from the viewpoint of their impact on food flavour. However, new studies on their antioxidant activity have been unraveled [8]. Fuster, Mitchell, Ochi \& Shibamoto [9] suggested that ingestion of heterocyclic compounds in roasted coffee and other products may be helpful in the prevention of in vivo oxidative damage such as lipid peroxidation which has been linked to diseases such as cancer, arteriosclerosis, aging, diabetes and immune deficiency. In addition, studies have reported antioxidant activity of certain volatile heterocyclic compounds (i.e. pyrroles, furans, thiophenes and thiazoles) in brewed coffee extracts $[5,10]$. Also, the antioxidant activities of aroma extracts from spices [11], herbs [12], and beans [13] have been reported.

However, to the best of our knowledge, the aroma chemistry and the contribution of the aroma compounds to the anti-oxidative properties of roasted yam has yet to be characterized. Therefore, the objectives of this study were; (1) to identify the key volatile compounds in roasted yam and (2). Evaluate the contributions of the selected volatiles to the anti-oxidative activity of roasted yam.

\section{Results and discussion}

\section{Aroma-active compounds in roasted yam}

Twenty-nine aroma-active compounds emanating from roasted yam were identified and quantified by $\mathrm{SH}-$ GC-MS and GC-O (Table 1). The compounds were made up of 16 aromatic compounds, 4 terpenes, 2 aldehydes, 3 ketones, 2 acids, 1 organic oxide ( $\beta$-cyclocitral) and a carboxylic ester (4-carbethoxybutyrolactone). The results of the $\mathrm{GC}-\mathrm{O}$ and AEDA revealed a flavour dilution (FD) factor range of 2-256 (Table 1). The aroma-active compounds produced an array of odour qualities such as toasty, caramel, popcorn-like, chamomile flower-like and smoky (Fig. 1). 2-Acetyl furan which elicited an almond-like note and 2-acetyl pyrrole with a popcorn-like note produced the highest FD factors of 128 and 256 respectively. Other compounds with significant FD factors $\geq 16$ were dihydro-2-methyl-3(2H) furanone (DHMF), 2-methylpyrazine, 2-ethylpyrazine, 5-methyl-2-furfural, 2-pentyl furan, ethyl furfural, phenyl acetaldehyde, 2-pyrrole carboxaldehyde, 2-ethyl-3,5-dimethyl pyrazine, 5-hydroxy methyl furfural and 4-carbethoxy butyrolactone. While there are no prior reports on the volatile flavour compounds of roasted yam in the literature, extensive research studies have been carried out on thermally processed potato tubers $[14,15]$. For instance, Dresow and Bohm [15] reported that compounds like 2-isobutyl-3-methoxypyrazine, 2-isopropyl3 -methoxypyrazine, $\beta$-damascenone, dimethyl trisulfide, decanal and 3-methylbutanal contributed significantly to baked potato flavour. In another study on baked 'jewel' sweet potatoes, Wang and Kays [16] established that phenyl acetaldehyde; maltol and methyl geranate possessed the highest FD factors (i.e. 1500). Whereas, a previous study on cooked water yam (Dioscorea alata) revealed that 4-phenyl butan-2-one (sweet/flowery) and alkymethoxypyrazine with characteristic green bell pepper note contributed majorly to the cooked water yam aroma [17]. In many respects the volatile compounds identified in the roasted yam were different from those observed as constituents of baked potatoes. The relative proportion of aromatic compounds and terpenes in roasted yam was significantly greater than in potatoes.

Aromatic compounds in roasted yam included 8 furans, 2 pyrroles, 3 pyrazines and 2 phenols (Table 1). Among these compounds, 2-acetyl furan, 2-pentyl furan, ethyl furfural, 5-methyl-2-furfural was reported as odor-active compounds in microwave-baked potatoes [18]. Furans have been identified in a number of food products especially in roasted and other thermally treated foods [19]. In addition, studies have proposed five different mechanisms for the formation of furans, namely: (1) thermal degradation of carbohydrates such as glucose, lactose and fructose; (2) the Maillard reaction between reducing sugars and amino acids; (3) the decomposition of ascorbic acid; (4) thermal oxidation of carotenoids; and (5) oxidation of polyunsaturated fatty acids [20-22].

The pyrroles and furanones are generated from sugar fragmentation of deoxyosones followed by reductions [23]. Pyrroles and furanones are classes of aroma-active compounds that have been reported extensively in coffee [24] but only few numbers of these compounds have been reported in potatoes. For instance, Oruma-Concha et al. [18] identified 2-methyl-1H-pyrrole and 2-methyl$3(2 \mathrm{H})$-furanone in baked potato. Similarly, $1 \mathrm{H}$-pyrrole2-carboxaldehyde has also been reported as constituent of many plants and roasted tea [25].

On the other hand, the pyrazines which normally elicits characteristic toasted, nutty and roasty notes in thermally processed foods are generally regarded as one of the most important aroma compounds in cooked flavour [26]. There are several precursors or pathways for pyrazine 
Table 1 Aroma-active compounds identified in roasted white yam (D. rotundata)

\begin{tabular}{|c|c|c|c|c|c|}
\hline No & Compounds $^{a}$ & Odour quality & RI on BPX5 & FD factor & GC peak area $\%^{b}$ \\
\hline 1 & Acetic acid & Vinegar & 600 & 4 & $0.25 \pm 0.00$ \\
\hline 2 & 3-Methyl butanal & Malty & 668 & 2 & $0.55 \pm 0.01$ \\
\hline 3 & Butanoic acid & Sweaty & 763 & 2 & $0.19 \pm 0.05$ \\
\hline 4 & Pentan-2-one & Ether-like & 711 & 4 & $0.10 \pm 0.01$ \\
\hline 5 & Dihydro-2-methyl-3(2H)-furanone & Toasty & 808 & 16 & $6.65 \pm 0.12$ \\
\hline 6 & 2-Methylpyrazine & Toasty & 820 & 64 & $8.87 \pm 0.78$ \\
\hline 7 & 3-Furaldehyde & Almond-like & 828 & 8 & $2.15 \pm 0.01$ \\
\hline 8 & 2-Furanmethanol & Caramel & 866 & 4 & $1.13 \pm 0.05$ \\
\hline 9 & 2-Acetylfuran & Cocoa/almond & 893 & 128 & $10.02 \pm 0.14$ \\
\hline 10 & 2-Ethylpyrazine & Nutty/roasty & 911 & 16 & $28.19 \pm 1.02$ \\
\hline 11 & Benzaldehyde & Almond-like & 960 & 8 & $4.17 \pm 0.01$ \\
\hline 12 & 5-Methyl-2-furfural & Spice/caramel & 978 & 16 & $20.68 \pm 0.11$ \\
\hline 13 & 2-Pentyl furan & Green bean & 993 & 16 & $12.54 \pm 0.33$ \\
\hline 14 & Ethyl furfural & Caramel/spice & 1020 & 64 & $13.29 \pm 1.58$ \\
\hline 15 & Limonene & Orange-like & 1033 & 4 & $0.58 \pm 0.00$ \\
\hline 16 & Phenyl acetaldehyde & Sweet rose & 1043 & 16 & $1.56 \pm 0.05$ \\
\hline 17 & 2-Acetylpyrrole & popcorn & 1045 & 256 & $18.31 \pm 0.04$ \\
\hline 18 & 2-Pyrrole carboxaldehyde & Musty/coffee & 1047 & 16 & $9.80 \pm 0.36$ \\
\hline 19 & 3-Methyl phenol & Phenolic/smoky & 1076 & 8 & $3.36 \pm 0.16$ \\
\hline 20 & 2-Ethyl-3,5-dimethylpyrazine & Earthy/roasty & 1088 & 16 & $4.03 \pm 0.03$ \\
\hline 21 & Linalool & Floral & 1100 & 4 & $0.52 \pm 0.01$ \\
\hline 22 & 5-Hydroxy methyl furfural & Chamomile flower-like & 1163 & 32 & $1.46 \pm 0.11$ \\
\hline 23 & $\beta$-Cyclocitral & Hay-like & 1218 & 2 & $0.10 \pm 0.01$ \\
\hline 24 & 2,6-Dimethoxyphenol & Smoky & 1349 & 2 & $0.45 \pm 0.01$ \\
\hline 25 & $\beta$-Caryophyllene & Woody/spice & 1408 & 8 & $1.62 \pm 0.11$ \\
\hline 26 & a-lonone & Floral & 1422 & 4 & $0.79 \pm 0.14$ \\
\hline 27 & a-Copaene & Woody & 1430 & 8 & $15.08 \pm 1.20$ \\
\hline 28 & $\beta$-lonone & Violet & 1493 & 2 & $1.29 \pm 0.01$ \\
\hline 29 & 4-Carbethoxybutyrolactone & Roasty/smoky & 1893 & 16 & $0.49 \pm 0.03$ \\
\hline
\end{tabular}

$R /$ retention index on BPX5 column, FD, flavour dilution

a Compounds were identified by comparing their retention indices on BPX 5 column, their mass spectra, and their odour notes with that of the respective reference standards

b Values are mean $\pm S D, n=3$

formation. The $\alpha$-amino carbonyls, which results from the reactions between dicarbonyl compounds and amino acids during Strecker degradation are generally considered to be the precursors of pyrazines [27]. Other classes of compounds identified in the roasted yam such as terpenes and phenols are not considered as potent odorants owing to their low $\mathrm{FD} \leq 8$ factors.

\section{Anti-oxidative activities of selected aroma compounds in roasted yam}

The inhibitory effect of the selected aroma compounds toward oxidative conversion of hexanal to hexanoic acid at five different concentrations was applied to evidence their anti-oxidative activities. The anti-oxidative activities of the selected aroma compounds are shown in Figs. 2, 3 and 4. The pyrroles (i.e. 2-pyrrole carboxaldehyde, and 2 -acetyl pyrrole) exhibited concentration-dependent activity and inhibited hexanal oxidation greatly (Fig. 2). For example, both 2-acetyl pyrrole and 2-pyrrole carboxaldehyde inhibited hexanal oxidation by approximately $100 \%$ at 50 fold their initial concentrations over 30 days (Table 2). In contrast, 2-pyrrole carboxaldehyde and 2-acetyl pyrrole, exhibited very low inhibition at $3.7 \mu \mathrm{g} \mathrm{mL}^{-1}$ and $1.68 \mu \mathrm{g} \mathrm{mL}^{-1}$ (being their concentrations in the roasted yam) over 30 days (Fig. 2). The antioxidative activities of pyrroles in coffee have been well documented $[5,28]$ and the ability of pyrroles to inhibit the oxidation of hexanal has been linked to the type of functional group attached to the pyrrole. Yanagimoto et al. [28] opined that the addition of electron-donating 


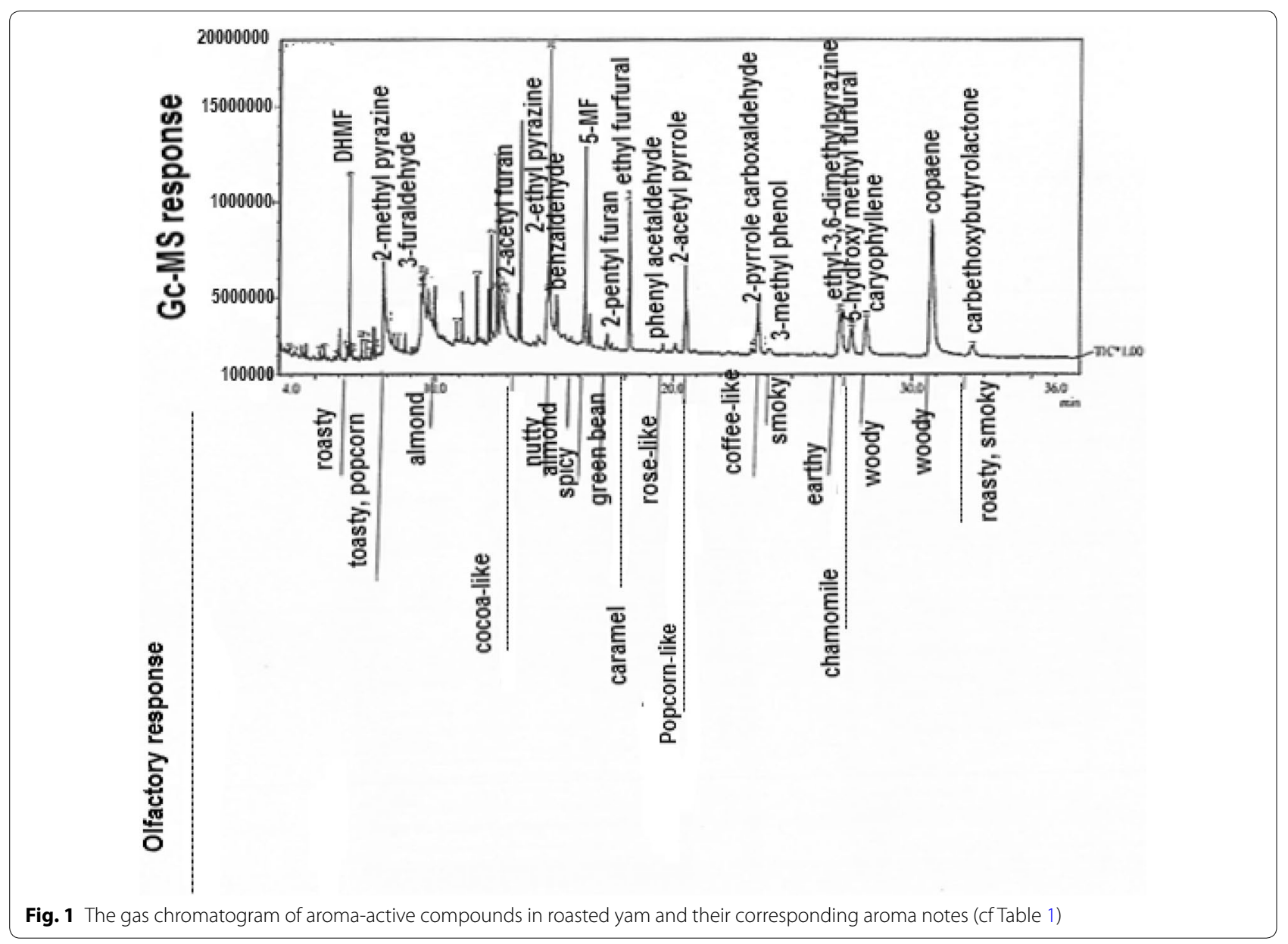

substituents such as methyl- and ethyl- to a heterocyclic ring increased radical scavenging ability of the pyrrole due to increase in the electron density at the carbon atoms. In converse, addition of electron-withdrawing functional group will reduce radical scavenging.

The furans also exhibited concentration-dependent activity (Fig. 3) similar to that of pyrroles. In the current study, 5-methyl-2-furfural inhibited hexanal oxidation by $8,56,60,65$ and $70 \%$ at levels of $8 \mu \mathrm{g} \mathrm{mL}^{-1}, 40 \mu \mathrm{g} \mathrm{mL}^{-1}$, $80 \mu \mathrm{g} \mathrm{mL}^{-1}, 161 \mu \mathrm{g} \mathrm{mL}^{-1}$ and $403 \mu \mathrm{g} \mathrm{mL}^{-1}$ over 30 days respectively (Table 2). Among the furans analyzed, 5-methyl-2-furfural exhibited the highest anti-oxidative activity (Fig. 3). This was followed by ethyl furfural and pentyl furan respectively. On the other hand, 2-acetyl furan had the least anti-oxidative activity. It inhibited hexanal oxidation by $20 \%$ at a level of $140 \mu \mathrm{g} \mathrm{mL}^{-1}$ (being 50 fold its concentration in the roasted yam). In the present study, the addition of the methyl-, ethyl- and pentylgroups greatly enhanced the anti-oxidative activity of the furan ring.

Pyrazines which are the most abundant heterocyclic constituents in non-enzymic browning reactions [7] exhibited only moderate hexanal oxidation inhibition in the roasted yam (Fig. 4). For instance, 2-ethylpyrazine inhibited hexanal oxidation by $20 \%$ over 30 days at a level of $47.5 \mu \mathrm{g} \mathrm{mL}^{-1}$ (i.e. 50 fold of its concentration in the roasted yam) (Table 2). Similarly, 2-methylpyrazine only inhibited hexanal oxidation by $30 \%$ at a level of $203 \mu \mathrm{g} \mathrm{mL}^{-1}$ for the same period. Earlier studies on roasted coffee have also reported low inhibition of pyrazines to hexanal oxidation [28-30]. For example, Yanagimoto et al. [28] reported that 2-methylpyrazine inhibited hexanal oxidation by $20 \%$ over 40 days at a level of $500 \mu \mathrm{g} \mathrm{mL}^{-1}$ in roasted coffee. In addition, they suggested that the addition of an electron-withdrawing group, such as an acetyl- group, to a pyrazine ring decreases anti-oxidative activity of the pyrazine while addition of electron-donating groups such as methyl- and ethyl- increases anti-oxidative activity [30]. The reason for this observation is because addition of electron-withdrawing groups to a heterocyclic ring decreases electron density around the ring and this result in decreased scavenging ability. In contrast, addition of electron-donating will increase radical scavenging [8]. However, in this 


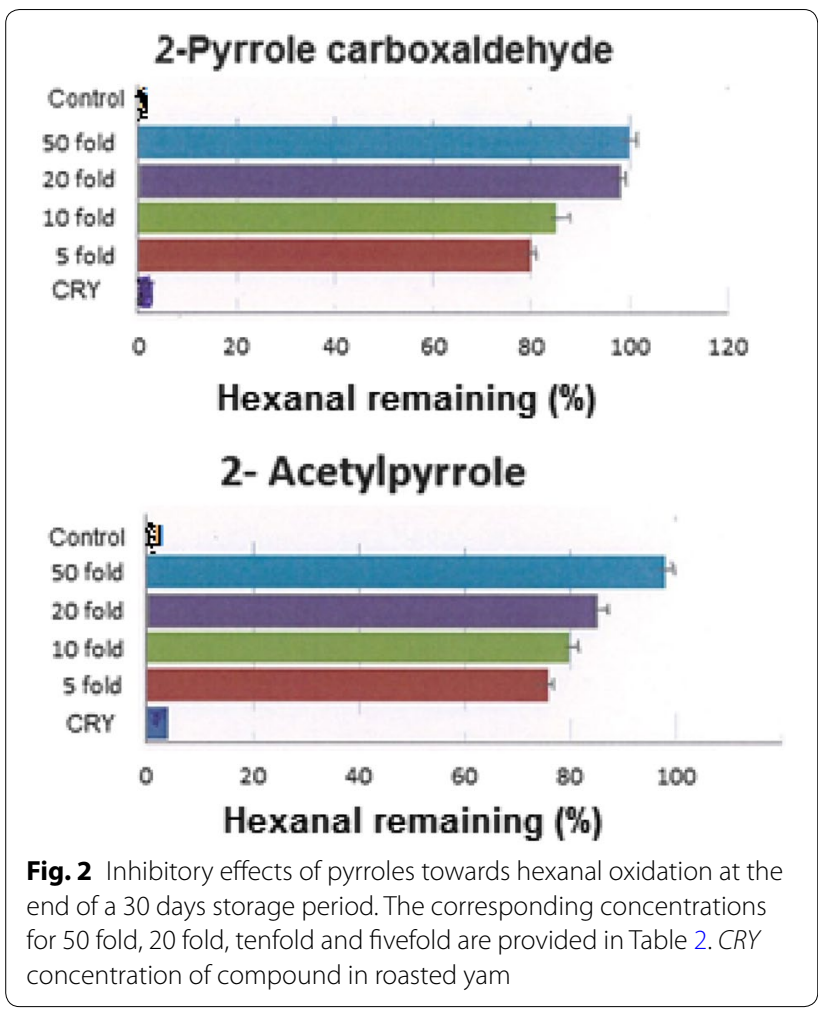

study, there was no significant increase in the anti-oxidative activities of 2-ethylpyrazine, 2-methylpyrazine and 2-ethyl-3,5-dimethyl pyrazine respectively. Therefore, it is possible that the effect of electron density of carbon atoms on a heterocyclic ring may not be the only contributing factor to the strength of anti-oxidative behaviour in pyrazines.

Whilst the levels of aroma compounds examined in this study were considerably higher than the actual levels found in the roasted yam, it is important to have a good knowledge of their anti-oxidative activities first in order to evaluate their activity at the more relevant low concentrations as obtained in the roasted yam. In addition the ingestion of the heterocyclic compounds may be helpful in preventing in vivo oxidative damage such as lipid peroxidation which has been linked to diseases like cancer, arteriosclerosis, aging, diabetes and immune deficiency [9].

\section{Conclusions}

The analysis of the aroma-active compounds of roasted yam by $\mathrm{SH}-\mathrm{GC}-\mathrm{MS}$ and $\mathrm{GC}-\mathrm{O}$ allowed the identification of 29 aroma-active compounds with FD factors within the range of 2-256 as the key aroma compounds in roasted yam. Among these are; 2-Acetyl furan which elicited an almond-like note and 2-acetyl pyrrole with a

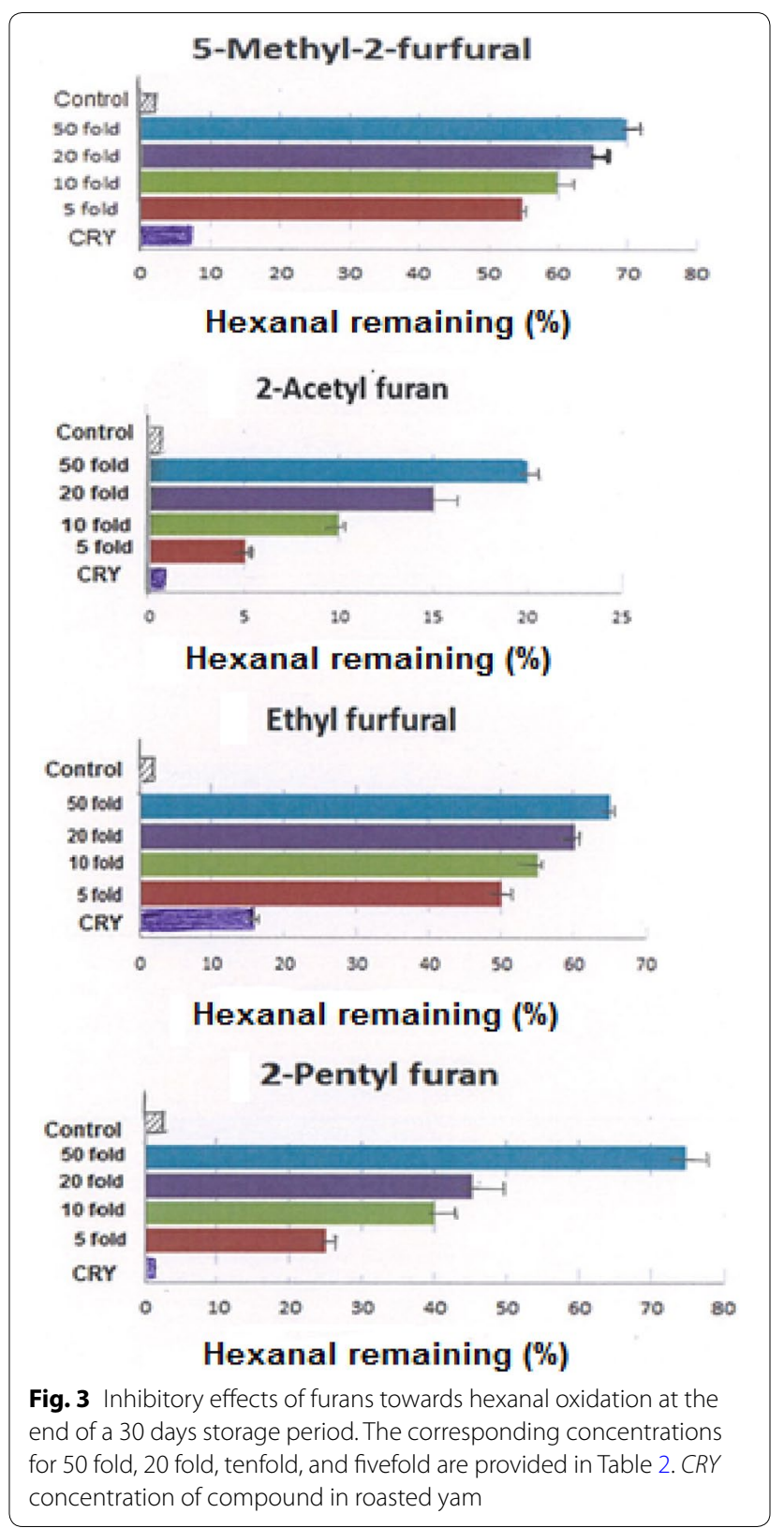

popcorn-like note produced the highest FD factors of 128 and 256 respectively. Other compounds with significant FD factors $\geq 16$ were dihydro-2-methyl-3(2H) furanone (DHMF), 2-methylpyrazine, 2-ethylpyrazine, 5-methyl2-furfural, 2-pentyl furan, ethyl furfural, phenyl acetaldehyde, 2-pyrrole carboxaldehyde, 2-ethyl-3,5-dimethyl pyrazine, 5-hydroxy methyl furfural and 4-carbethoxy butyrolactone. In addition, varying degrees of anti-oxidative activities were obtained from the selected volatile heterocyclic compounds ( 2 pyrroles, 4 furans and 3 pyrazines). Pyrroles exhibited the greatest oxidative activity followed by the furans and the pyrazines with 


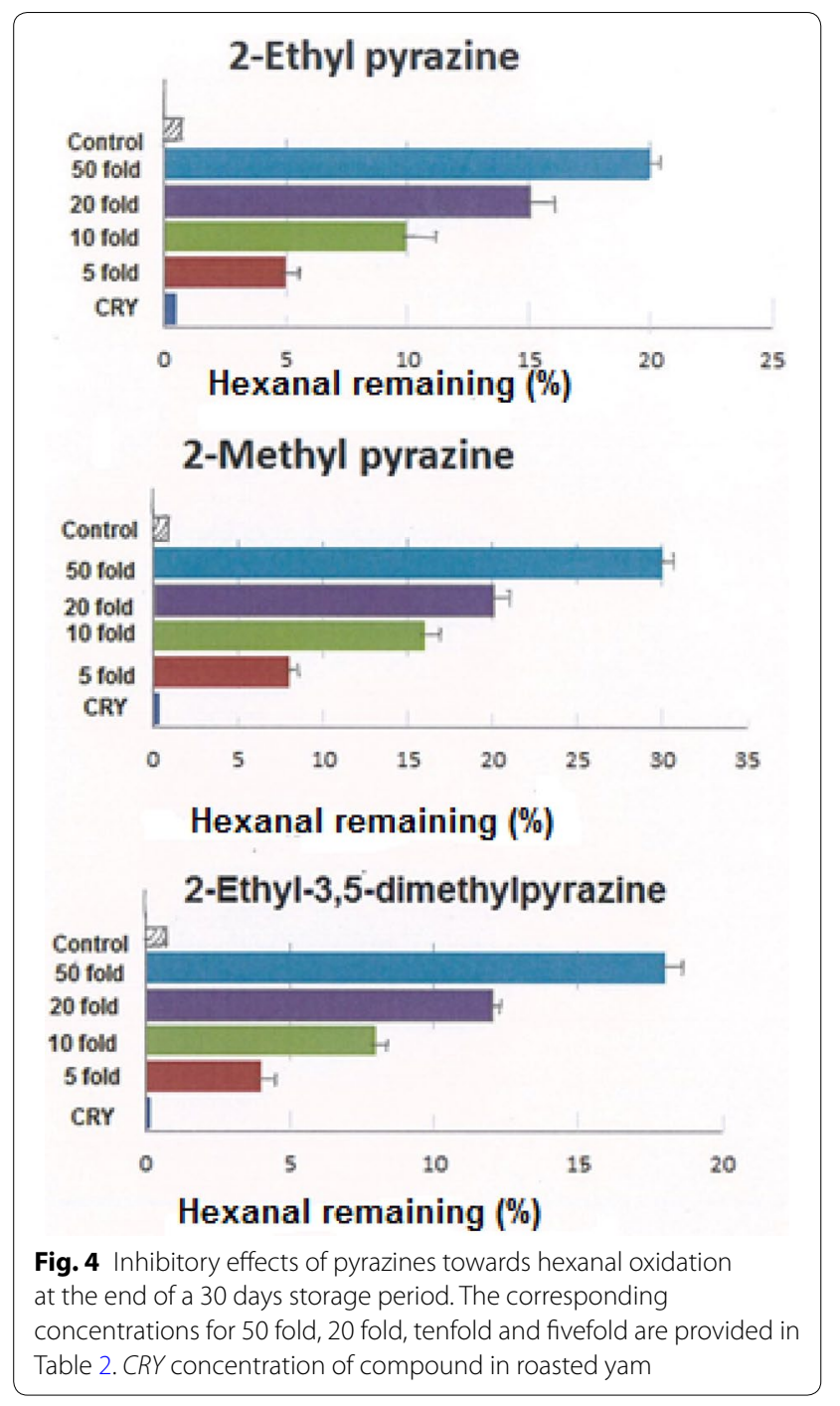

the least activity. The pyrroles inhibited hexanal oxidation by almost $100 \%$ at 50 fold of their concentrations in the roasted yam over 30 days of storage. The pyrazines exhibited very low anti-oxidative activity throughout the 30 days storage. Moreover, the addition of functional groups found in Maillard reaction products such as acetyl-, ethyl-, and methyl-groups influenced the antioxidative activities of the heterocyclic compounds.

\section{Experimental \\ Chemicals}

The necessary chemical standards were obtained from the suppliers shown in parenthesis. Acetic acid, 99\% (Merck, Darmstadt, Germany); 3-methyl butanal, 98\%; butanoic acid, 97\%; pentan-2-one, 98\%; dihydro-2-methyl-3(2H) furan, 97\%; 2-methylpyrazine, 98\%; 3-furaldehyde, 99\%; 2-furamethanol, 97\%; 2-acetyl furan, 99\%; 2-ethylpyrazine, 98\%; benzaldehyde, 98\%; 5-methyl2-furfural, 99\%; 2-pentyl furan, 98\%; ethyl furfural, 98\%; limonene, 99\%; phenyl acetaldehyde, 99\%; 2-acetylpyrrole, 98\%; 2-pyrrole carboxaldehyde, 97\%; 3-methyl phenol, 99\%; 2-ethyl-3,5-dimethylpyrazine, 98\%; linalool, 98\%; 5-hydroxy methyl furfural, 99\%; $\beta$-cyclocitral, 97\%; 2,6-dimethoxyphenol, 98\%; $\beta$-caryophyllene, 98\%; $\alpha$-ionone, 98\%; $\alpha$-copaene, 99\%; $\beta$-ionone, 98\% (Aldrich, Steinheim, Germany).

\section{Preparation of roasted yam tubers}

Yam tubers (Discorea rotundata; elongated with thick rough hairy skin) (500 g) each were purchased from Pasar Borong, Selangor, Malaysia and were identified by Prof. Mohd Yusop of the Faculty of Agriculture, Malaysia.. The yam samples were stored at $4 \pm 1{ }^{\circ} \mathrm{C}$ until used. Yam tubers with uniform size, maturity (i.e. yam harvested

Table 2 Concentrations $\left(\mu \mathrm{g} \mathrm{mL}^{-1}\right.$ ) of the selected aroma-active compounds in roasted white yam (Dioscorea rotundata)

\begin{tabular}{|c|c|c|c|c|c|c|}
\hline No & Compound & Conc. In roasted yam & $\begin{array}{l}\text { Fivefold conc. } \\
\text { In yam }\end{array}$ & $\begin{array}{l}\text { Tenfold conc. } \\
\text { In yam }\end{array}$ & $\begin{array}{l}20 \text { fold conc. } \\
\text { In yam }\end{array}$ & $\begin{array}{l}50 \text { fold conc. } \\
\text { In yam }\end{array}$ \\
\hline \multicolumn{7}{|c|}{ Furans } \\
\hline 1 & 2-Acetyl furan & $2.80 \pm 0.01$ & 14.0 & 28.0 & 56.0 & 140.0 \\
\hline 2 & 5-Methyl-2-furfural & $8.06 \pm 0.23$ & 40.3 & 80.6 & 161.2 & 403.0 \\
\hline 3 & 2-Pentyl furan & $2.56 \pm 0.10$ & 12.8 & 25.6 & 51.2 & 128.0 \\
\hline 4 & Ethyl furfural & $16.23 \pm 0.45$ & 81.2 & 162.3 & 324.6 & 811.5 \\
\hline \multicolumn{7}{|c|}{ Pyrroles } \\
\hline 1 & 2-Acetylpyrrole & $1.68 \pm 0.01$ & 8.4 & 16.8 & 33.6 & 84.0 \\
\hline 2 & 2-Pyrrole carboxaldehyde & $3.71 \pm 0.02$ & 18.6 & 37.1 & 74.2 & 185.5 \\
\hline \multicolumn{7}{|c|}{ Pyrazines } \\
\hline 1 & 2-Ethylpyrazine & $0.95 \pm 0.01$ & 4.75 & 9.5 & 19.0 & 47.5 \\
\hline 2 & 2-Methylpyrazine & $4.06 \pm 0.12$ & 20.3 & 40.6 & 81.2 & 203 \\
\hline 3 & 2-Ethyl-3,5-dimethylpyrazine & $0.70 \pm 0.01$ & 3.5 & 7.0 & 14.0 & 35.0 \\
\hline
\end{tabular}


220 days after planting, DAP) and free from defects were selected, washed and hand peeled to remove the skin and roasted $\left(400{ }^{\circ} \mathrm{C}\right.$ for $80 \mathrm{~min}$ ) in a roaster (Model Duetl$\mathrm{M}$, Probat and Emmerich, Germany). After roasting, the yam tubers were removed from the oven and allowed to cool to room temperature $\left(28 \pm 1{ }^{\circ} \mathrm{C}\right)$ prior to solvent extraction.

\section{Yam extract production}

A longitudinal section cut (50 g of each roasted yam tubers) obtained from the central part was pulverized and a portion of the pulverized roasted yam (10 g) was taken for volatile compounds analysis. Aroma extract was prepared from a $10 \mathrm{~g}$ of the pulverized roasted tubers with a $40 \mathrm{~mL}$ of dichloromethane. The suspension was stirred using a vortex mixer (Heidolph, Rotamax 120, Schwabach, Germany) at $150 \mathrm{rpm}$ and $28^{\circ} \mathrm{C}$ for $1 \mathrm{~h}$. The extract was filtered and dried on $\mathrm{Na}_{2} \mathrm{SO}_{4}$. The solvent was subsequently removed under a purified nitrogen stream (Turbo Vap II, Caliper Life Science, Massachusetts, USA) to a volume of $4 \mathrm{~mL}$. The extract was stored at $-20{ }^{\circ} \mathrm{C}$ until analysis.

\section{Analysis of volatile compounds}

Analysis of volatiles was carried out using the Static headspace-gas chromatography-mass spectrometry ( $\mathrm{SH}-$ GC-MS) as reported by Ludwig, et al. [5]. The roasted extract $(4 \mathrm{~mL})$ or volatile standards solution $(4 \mathrm{~mL})$ was introduced into a $10 \mathrm{~mL}$ vial and sealed with a silicon rubber Teflon cap. The vial was equilibrated $\left(40{ }^{\circ} \mathrm{C}\right.$, $15 \mathrm{~min}$ ) in the headspace sampler (model $7694 \mathrm{E}$, Agilent Technologies, Palo Alto, CA) with pressurized carrier gas for $12 \mathrm{~s}$. Subsequently, $1 \mathrm{~mL}$ of the headspace was injected into a non-polar BPx5 (5\% phenyl polysilylphenylene siloxane) capillary column $(30 \mathrm{~m} \times 0.25 \mathrm{~mm}$ i.d., film thickness $0.25 \mu \mathrm{m}$; Scientific Instrument Services, Inc., Ringoes, NJ. USA) in a QP-5050A GC-MS Instrument (Shimadzu, Kyoto, Japan). Helium at a flow rate of $1.5 \mathrm{~mL} \mathrm{~min}{ }^{-1}$, injection temperature at $250{ }^{\circ} \mathrm{C}$ and a detector temperature of $280{ }^{\circ} \mathrm{C}$ were employed. The temperature program commenced at $50{ }^{\circ} \mathrm{C}$ and was held for $3 \mathrm{~min}$, raised to $250{ }^{\circ} \mathrm{C}$ at the rate of $15{ }^{\circ} \mathrm{C} \mathrm{min}{ }^{-1}$, held for $30 \mathrm{~min}$ and later increased to $280{ }^{\circ} \mathrm{C}$ at a rate of $10^{\circ} \mathrm{C}$ $\mathrm{min}^{-1}$, with a final hold time of $5 \mathrm{~min}$.

Mass spectrometry was carried out in electron impact mode using the following conditions: The source temperature was $250{ }^{\circ} \mathrm{C}$, the quadruple temperature selected was $280{ }^{\circ} \mathrm{C}$ and the relative electron multiplier voltage applied was $400 \mathrm{~V}$ with a resulting voltage of $1553 \mathrm{~V}$. The selection ion monitoring mode was employed to improve the detection limits. Identification of volatile compounds (Table 1) was done comparing their mass spectra with the spectra of the available pure standards and also by comparing their retention indices with those of standards and data from literature. The linear retention indices (RI) of compounds were obtained with a series of alkanes $\left(\mathrm{C}_{6}-\right.$ $\mathrm{C}_{28}$ ) injected according to the same chromatographic protocol reported above.

\section{Gas-chromatography-olfactometry analysis}

The GC-O system was made up of a Trace Ultra 1300 GC (Thermos Scientific, Waltham, MA, USA) equipped with an ODP 3 olfactory detector port (Gerstel, Mulheim, Germany). A BPX5 capillary column $(30 \mathrm{~m} \times 0.25 \mathrm{~mm}$ i.d., film thickness $0.25 \mu \mathrm{m}$; Scientific Instrument Service, Inc., Ringoes, NJ, USA) was employed. The column temperature program was similar to that of the GC-MS above. The flow rate was $1 \mathrm{~mL} \min ^{-1}$ and $1 \mu \mathrm{L}$ of the yam extract was injected. The split ratio between the FID detector and the sniffing port was 1:1. Sniffing was conducted through a sniffing cone by three trained panellists that presented normalized responses.

Aroma extract dilution analysis (AEDA) was employed in establishing the contribution of each aroma compound to the overall flavour of the roasted yam. The extract was diluted stepwise with dichloromethane $(1: 1, \mathrm{v} / \mathrm{v})$ and sniffing of dilutions was continued until no odour could be detected by sniffers [31]. The last dilution step in which an aroma compound was sniffed is referred to as the flavour dilution (FD) factor of the compound [32].

\section{Anti-oxidative activity of volatile compounds}

The anti-oxidative activities of the selected aroma compounds were tested according to the method reported by Yangimoto et al. [28]. Nine volatile compounds (4 furans, 2 pyrroles, and 3 pyrazines) with appreciable concentrations in the roasted yam (Table 2) and previously identified as potential anti-oxidants in roasted coffee [5] were further quantified by calibration curves to determine their concentrations in the roasted yam. Standard solutions of each selected volatile compound were analysed at five levels of concentrations and their chromatographic areas were plotted against concentration. The coefficients of linearity for the calibration curves were $\mathrm{R}^{2}>0.98$. Pure reference standards at concentrations equivalent to those of the selected volatiles found in the roasted yam and at $5,10,20$, and 50 fold of the initial concentration found in the roasted yam (Table 2) were prepared. Each reference standard was added to $2 \mathrm{~mL}$ of dichloromethane solution of hexanal $\left(3 \mathrm{mg} \mathrm{mL}^{-1}\right)$ containing $0.2 \mathrm{mg} \mathrm{mL}^{-1}$ of undecane (internal standard) in a $20 \mathrm{~mL}$ vial. The sealed vial was heated at $60{ }^{\circ} \mathrm{C}$ for $10 \mathrm{~min}$ to initiate oxidation reaction of the mixture within the sealed vial. Analysis of inhibition of aroma compounds toward hexanal was carried out by purging the headspace of the vial with pure 
air $\left(1.5 \mathrm{~L} \mathrm{~min}{ }^{-1}, 2 \mathrm{~s}\right)$ every $24 \mathrm{~h}$ for the first 10 days. The inhibition of aroma compounds toward hexanal was monitored by gas chromatography (GC) at 5-days interval for 30 days.

\section{Abbreviations}

MRPs: Maillard reaction products; AGEs: amines acryl amines; SH-GC-MS: static-headspace-gas chromatography-mass spectrometry; AEDA: aroma extract dilution analysis; FD: flavour dilution.

\section{Acknowledgements}

Not applicable.

\section{Authors' contributions}

OL Conceptualized designed the research and corrected the final manuscript; LST performed the experimental work and analyzed the data and prepared the manuscript. Both authors read and approved the final manuscript.

\section{Funding}

This research was funded by the Research University Grant Scheme (Jenis Geran. No. 9478500) of the University Putra Malaysia, UPM, 43400 Serdang, Malaysia.

\section{Availability of data and materials}

The dataset generated and/or analysed during this study cannot be released now because the research is still on going. However, the data are available on request from the corresponding author.

\section{Competing interests}

The authors declare that they have no competing interests.

Received: 26 June 2019 Accepted: 8 November 2019

Published online: 19 December 2019

\section{References}

1. Price EJ, Wilkin P, Sarasan V, Fraser PD (2016) Metabolite profiling of Dioscorea (yam) species reveals underutilised bioderversity and renewable sources for high-value compounds. Sci Rep 6:29136. https://doi. org/10.1038/srep29136

2. Mignouna HD, Abang MM, Asiedu R (2003) Harnessing modern biotechnology for tropical tuber crop improvement: Yam (Dioscorea spp.) molecular breeding. Afr J Biotechnol 2:478-485

3. Lasekan O, Khatib A, Juhari H, Patiram P, Lasekan A (2012) High-performance liquid chromatographic determination of 5-hydroxymethyl furfural in roasted plantain cultivars. J Food Agric Environ 10:99-103

4. Qin G, Tao S, Cao Y, Wu J, Zhang H, Huang W, Zhang S (2012) Evaluation of the volatile profile of 33 Pyrus ussuriensis cultivars by HS-SPME with GC-MS. Food Chem 134:2367-2382

5. Ludwig IA, Sanchez L, DePena MP, Cid C (2014) Contribution of volatile compounds to the antioxidant capacity of coffee. Food Res Int 62:67-74

6. Wang HY, Qian H, Yao WR (2011) Melanoidins produced by the Maillard reaction: structure and biological activity. Food Chem 128:573-584

7. Shibamoto $T$ (1983) Heterocyclic compounds in brewing and browning/ nitrite model Occurrence, formation-mechanisms, flavour characteristics and mutagenic activity. In: Charalambous G, Inglett G (eds) Instrumental Analysis of Foods. Academic Press, New York, pp 229-278

8. Eiserich JP, Wang JW, Shibamoto T (1995) Anti-oxidative activities of furans and thiophenethiols measured in lipid peroxidation systems and tyrosyl radical scavenging assay. Journal of Agricultural and Food Chemistry 43:647-650

9. Fuster MD, Mitchell AE, Ochi H, Shibamoto T (2000) Antioxidative activities of heterocyclic compounds formed in brewed coffee. Journal of Agricultural and Food Chemistry 48:5600-5603

10. Delgado-Andrade C, Rufian-Henares JA, Morales FJ (2005) Assessing the antioxidant activity of melanoidins from coffee-brews by different antioxidant methods. Journal of Agricultural and Food Chemistry 53:7832-7836
11. Nakatami N (1997) Antioxidants from spices and herbs. In: Shahidi F (ed) Natural antioxidants: chemistry, health effects and applications. Illinois AOAC Press, Champaign, pp 64-75

12. Okuda T (1999) Antioxidants in herbs; Polyphenols. In: Packer L, Hiramatsu M, Yoshikawa T (eds) Antioxidant food supplements in human health. Academic Press, San Diego, pp 393-410

13. Lee KC, Mitchell AE, Shibamoto T (2000) Determination of antioxidant properties of aroma extracts isolated from various beans. Journal of Agricultural and Food Chemistry 48:4817-4820

14. Duckham SC, Dodson AT, Bakker J, Ames JM (2001) Volatile flavour components of baked potato flesh: a comparison of eleven potato cultivars. Nahrung 45:317-323

15. Dresow JF, Bohm H (2009) The influence of volatile compounds of the flavour of raw boiled and baked potatoes: impact of agricultural measures on the volatile components. Landbaurforschung-VTI Agriculture and Forestry Research 4:309-333

16. Wang Y, Kays SJ (2000) Contribution of volatile compounds to the characteristic aroma of baked 'jewel' sweet potatoes. Journal of American Society of Horticultural Science 125:638-643

17. Gramshaw JW, Osinowo FAO (1982) Volatile components of cooked tubers of water yam (Dioscorea alata). Journal of Science and Food Agriculture 33:71-80

18. Oruma-Concha MJ, Bakker J, Ames JM (2002) Comparison of the volatile components of eight cultivars of potato after microwave baking. LWTFood Science and Technology 35:80-86

19. Fromberg A, Mariotti MS, Pedreschi F, Fagt S, Granby K (2014) Furan and alkylated furans in heat processed food including home cooked products. Czech Journal of Food Science 32:443-448

20. Yaylayan VA (2006) Precursors, formation and determination of furan in food. Journal For Consumer Protection and Food Safety 1:5-9

21. Perez-Lucas C, Yaylayan VA (2004) Origin and mechanistic pathways of formation of the parent furan: a food toxicant. Journal of Agricultural and Food Chemistry 52:6830-6836

22. Becalski A, Seman S (2005) Furan precursors in food: a model study and development simple headspace method for determination of furan. J AOAC Int 88:102-106

23. Hertz-Schunemann R, Streibel T, Ehlert S, Zimmermann R (2013) Looking into individual coffee beans during the roasting process: direct microprobe sampling on-line photo-ionisation mass spectrometric analysis of coffee roasting gases. Anal Bioanal Chem 405:7083-7096

24. Zapata J, Londono V, Naranjo M, Osorio J, Lopez C, Quintero M (2018) Characterization of aroma compounds present in an industrial recovery concentrate of coffee flavour. CyTA- Journal of Food 16:367-372

25. Sheibani E, Duncan SE, Kuhn DD, Dietrich AM, Newkirk JJ, O'Keefe SF (2016) Changes in flavour volatile composition of oolong tea after panning during tea processing. Food Science and Nutrition 4:456-468

26. Maga JA (1992) Pyrazine update. Food Res Int 8:479-558

27. Yu AN, Zhang AD (2010) The effect of $\mathrm{pH}$ on the formation of aroma compounds produced by heating a model system containing L-ascorbic acid with L-threonine/L- serine. Food Chem 119:214-219

28. Yanagimoto K, Lee KG, Ochi H, Shibamoto T (2002) Anti-oxidative activity of heterocyclic compounds found in coffee volatiles produced by Maillard reaction. Journal of Agricultural and Food Chemistry 50:5480-5484

29. Yanagimoto K, Ochi H, Lee KG, Shibamoto T (2004) Antioxidative activities of fractions obtained from brewed coffee. Journal of Agricultural and Food Chemistry 52:592-596

30. Osada Y, Shibamoto T (2006) Antioxidative activity of volatile extracts from Maillard model systems. Food Chem 98:522-528

31. Lasekan O, Muniandy M, Lin MG, Dabaj F (2018) Identification of characteristic aroma compounds in raw and thermally processed African giant snail (Achatina fulica). Chem Cent J 12:43-50

32. Schieberle P (1995) Recent developments in methods for analysis of flavour compounds and their precursors. In: Gaonkar A (ed) Characterization of food: emerging methods. Elsevier, Amsterdam, pp 403-431

\section{Publisher's Note}

Springer Nature remains neutral with regard to jurisdictional claims in published maps and institutional affiliations. 